\title{
DYNAMIC TIME WARPING ANALYSIS IN POST-STROKE REHABILITATION
}

\author{
Bálint Magyar ${ }^{1}$, Gábor Stépán ${ }^{1}$, I-Ming Chen ${ }^{2}$ \\ ${ }^{1}$ Budapest University of Technology and Economics, Faculty of Mechanical Engineering, \\ Department of Applied Mechanics \\ ${ }^{2}$ Nanyang Technical University, School of Mechanical and Aerospace Engineering \\ magyar@mm.bme.hu
}

\begin{abstract}
This paper presents the application of the Dynamic Time Warping (DTW) algorithm in the analysis of human functional movements in activities of daily living (ADLs). Dynamic Time Warping was originally developed for automatic speech recognition, though the method has been adopted by several fields of biomechanics. As a part of the post-stroke rehabilitation project COSMOSYS, the aim is to quantify the ADL performances of hemiparetic subjects, hence to be able to track their progress during physiotherapy.
\end{abstract}

Keywords: dynamic time warping, stroke rehabilitation

\section{Introduction}

Dynamic Time Warping in automatic speech recognition is used to measure the similarity of two audio sequences, which may vary in time and speed. The sequences are warped nonlinearly in the time dimension to determine the ,score” of their similarity independent of certain non-linear variations in pace. The sequences are warped non-lin-early in the time dimension to determine the "score" of their similarity independent of cer-tain non-linear variations in pace for various applications of DTW. ${ }^{1-6}$ The rehabilitation of hemiparesis after stroke demanded a comparison method that is able to express the correlation of two data sets. The aim is to evaluate the measured human functional movements also called Activities of Daily Living (ADLs), i.e. to qualify those with a single scalar. The difference of the measured and the reference ADL data sets can be used to evaluate the patients' performance.
The present approach bridges the gap between the objective sensory information available on normal and pathological human movements on one side and the subjective qualitative evaluation of these motions by the skilled professional in the form of performance scales on the other side. While the latter have already been standardized among clinical professionals, the error of human motion cognition by objective evaluation is still remarkable: evaluation of human movements may differ due to the imperfection of human cognitive capabilities, or simply from training, practice, institution, location and nationality of the clinical professional. The error can be radically reduced by the proposed co-iterative analytical-statistical method. Feeding of the DTW metrics into the robot controller makes the robot driven physiotherapy biomedically determined. In present approach the DTW metrics have been produced from measurements by $3 \mathrm{D}$ motion analyser whereas the robot can measure the same parameters from their integrated sensors: the motor encoders. 


\section{ADL Measurements}

The ADLs to be measured on both healthy and post-stroke patients were selected by therapists of the Hungarian National Institute for Medical Rehabilitation, and the Singaporean Tan Tock Seng Hospital.

These ADLs are the following:

- picking up a phone from the table and placing it back;

- picking up a mug by its handle, moving it to the mouth and placing it back onto the table; - opening and closing a window by its handle; - picking up a towel, moving it to the face and placing it back;

- doing and undoing a zipper on a vest.

\section{Measurement Device}

The motion capture device, shown in Figure 1, consists of the Smartsuit and the Smartglove, developed in Nanyang Technological University, Singapore. Smartsuit is an IMU (Inertial Measurement Unit) based motion analyser for measuring upper arm and lower arm orientations. Smartglove acquires finger flexion-

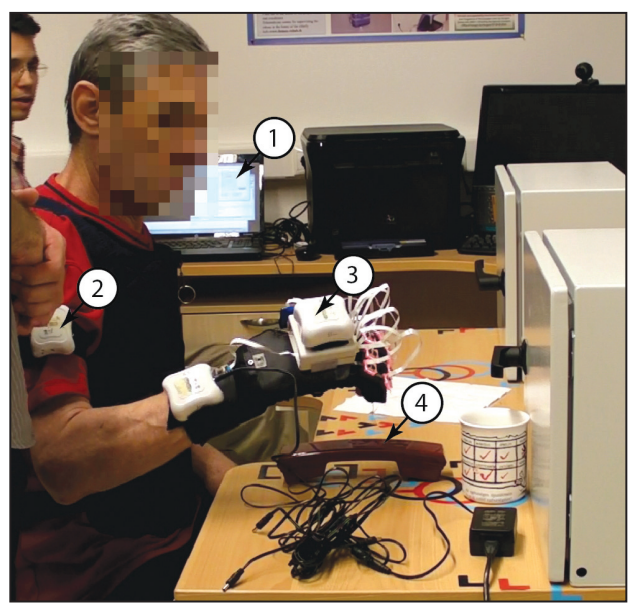

Figure 1. ADL measurement

1: data acquisition software; 2: Smartsuit; 3: Smartglove; 4: ADL items extension and palm orientation, by using optical encoders and an IMU. Finger flexionextension data were excluded in the data mining analysis.

The pose of the hand is described by the feature vector $\mathbf{f}$, that is

$\mathbf{f}=\left(\alpha_{\mathrm{s}}, \beta_{\mathrm{s}}, \gamma_{\mathrm{s}}, \beta_{\mathrm{e}}, \alpha_{\mathrm{w}}, \beta_{\mathrm{w}}, \gamma_{\mathrm{w}}\right)^{\mathrm{T}}$

which contains the anatomic angles of the shoulder, $e$ lbow and the $w$ rist. The definitions of these anatomic angles were inherited from the "Jack" human simulation software by Siemens PLM. The three IMUs in the Smartsuit and Smartglove provide the spatial orientations (roll, pitch, yaw) directly for the upper arm, lower arm and palm, while anatomic angles can be calculated afterwards. Figure 2 illustrates the coordinate system and the reference points (S, E and $\mathrm{W}$ ) on the arm. For a given length of the upper $\operatorname{arm}\left(L_{\mathrm{UA}}\right)$, lower $\operatorname{arm}\left(L_{\mathrm{LA}}\right)$ and palm $\left(L_{\mathrm{P}}\right)$, the coordinates of the reference points can be calculated as follows:

$$
\begin{aligned}
& \mathbf{r}_{\mathrm{SE}}=\mathbf{T}_{\mathrm{y}}^{-\beta_{\mathrm{s}}} \mathbf{T}_{\mathrm{x}}^{\alpha_{\mathrm{s}}} \mathbf{T}_{\mathrm{z}}^{-\gamma_{\mathrm{s}}} \mathbf{r}_{\mathrm{SE}}^{0}, \\
& \mathbf{r}_{\mathrm{SW}}=\mathbf{T}_{\mathrm{y}}^{-\beta_{\mathrm{s}}} \mathbf{T}_{\mathrm{x}}^{\alpha_{\mathrm{s}}} \mathbf{T}_{\mathrm{z}}^{-\gamma_{\mathrm{s}}}\left(\mathbf{T}_{\mathrm{y}}^{-\beta_{\mathrm{e}}} \mathbf{r}_{\mathrm{EW}}^{0}+\mathbf{r}_{\mathrm{SE}}^{0}\right),
\end{aligned}
$$

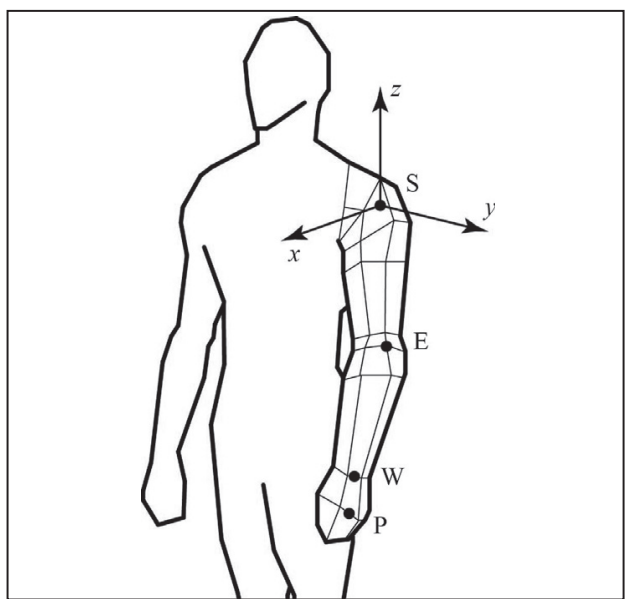

Figure 2. Base coordinate system and reference points on the human arm 
$\mathbf{r}_{\mathrm{SP}}=\mathbf{T}_{\mathrm{y}}^{-\beta_{\mathrm{s}}} \mathbf{T}_{\mathrm{x}}^{\alpha_{\mathrm{s}}} \mathbf{T}_{\mathrm{z}}^{-\gamma_{\mathrm{s}}}\left(\mathbf{T}_{\mathrm{y}}^{-\beta_{\mathrm{e}}}\left(\mathbf{T}_{\mathrm{z}}^{-\beta_{\mathrm{w}}} \mathbf{T}_{\mathrm{x}}^{\alpha_{\mathrm{w}}} \mathbf{T}_{\mathrm{y}}^{-\gamma_{\mathrm{w}}} \mathbf{r}_{\mathrm{WP}}^{0}+\right.\right.$,

$\left.\left.+\mathbf{r}_{\mathrm{EW}}^{0}\right)+\mathbf{r}_{\mathrm{SE}}^{0}\right)$

where $\mathbf{r}_{\mathrm{SE}}^{0}=\left(0,0,-L_{\mathrm{UA}}\right)^{\mathrm{T}}, \mathbf{r}_{\mathrm{EW}}^{0}=\left(0,0,-L_{\mathrm{LA}}\right)^{\mathrm{T}}$, $\mathbf{r}_{\mathrm{WP}}^{0}=\left(0,0,-L_{\mathrm{P}}\right)^{\mathrm{T}}$, with superscript 0 referring to the initial state, and $\mathbf{T}_{\mathrm{x}}^{\alpha}, \mathbf{T}_{\mathrm{y}}^{\beta}, \mathbf{T}_{\mathrm{z}}^{\gamma}$ are the rotation matrices with superscripts referring to the angle of rotation about the corresponding axes noted in the subscripts.

\section{Dynamic Time Warping}

A wide-spread method for computing the similarity between two time series is the Dynamic Time Warping algorithm, which is based on the alignment of significant patterns by locally deforming the time axis (hence the name warping) in order to minimize the cumulative difference between the aligned points. In order to obtain the optimal alignment, local compressions and shifts are achieved, thus, the method is suitable for matching time series containing patterns that are qualitatively similar but have different lengths and paces. ${ }^{1}$

\section{Definitions}

The inputs of the DTW algorithm are two data sequences, not necessarily containing the same number of samples. Let these sequences be noted by $\mathbf{F}^{\mathrm{I}}=\left(\mathbf{f}_{1}^{\mathrm{I}}, \ldots, \mathbf{f}_{i}^{\mathrm{I}}, \ldots, \mathbf{f}_{n}^{\mathrm{I}}\right)$ and $\mathbf{F}^{\mathrm{II}}=\left(\mathbf{f}_{1}^{\mathrm{II}}, \ldots, \mathbf{f}_{j}^{\mathrm{II}}, \ldots, \mathbf{f}_{m}^{\mathrm{II}}\right)$, where $\mathbf{f}_{i}^{\mathrm{I}}, \mathbf{f}_{j}^{\mathrm{II}} \in \mathbb{R}^{q}$ for $i \in[1, n], j \in[1, m]$ and $q$ is the dimension of the feature space. The outputs are the warping curve $\left(\Phi^{\mathrm{I}}, \Phi^{\mathrm{II}}\right)$ and the DTW distance $\delta$, where $\Phi^{\mathrm{I}}=\left(\phi_{1}^{\mathrm{I}}, \ldots, \phi_{k}^{\mathrm{I}}, \ldots, \phi_{K}^{\mathrm{I}}\right)$ and $\Phi^{\mathrm{II}}=\left(\phi_{1}^{\mathrm{II}}, \ldots\right.$, $\left.\phi_{k}^{\mathrm{II}}, \ldots, \phi_{K}^{\mathrm{II}}\right)$. These vectors contain the sample indices of $\mathbf{F}^{\mathrm{I}}$ and $\mathbf{F}^{\mathrm{II}}$ that should be selected to obtain the optimal fit $\left(\Phi^{\mathrm{I}}, \Phi^{\mathrm{II}} \in \mathbb{Z}^{\mathbf{K}}\right)$. In order to calculate the warping curve, the constrained minimization problem

$$
\left(\Phi^{\mathrm{I}}, \Phi^{\mathrm{II}}\right)=\arg \min _{\Psi^{\mathrm{I}}, \Psi^{\mathrm{II}}} \sum_{k=1}^{K} \mathrm{D}\left(\mathbf{f}_{\phi_{k}^{\mathrm{I}}}^{\mathrm{I}}, \mathbf{f}_{\phi_{k}^{\mathrm{II}}}^{\mathrm{II}}\right)
$$

should be solved where $\mathrm{D}$ is the scalar-valued penalizing function that represents the distance between two feature vectors as detailed in (1). Several different sets of constraints can be found in the literature ${ }^{1}$ for the warping curve $\left(\Phi^{\mathrm{I}}, \Phi^{\mathrm{II}}\right)$. In this study, the following set is applied:

- asymmetric start-point constraint: $\phi_{1}^{\mathrm{I}}=1$ and/or $\phi_{1}^{\mathrm{II}}=1$,

- end-point constraint: $\phi_{K}^{\mathrm{I}}=n$ and $\phi_{K}^{\mathrm{II}}=m$,

- monotonicity: $\phi_{k+1}^{\mathrm{I}} \geq \phi_{k}^{\mathrm{I}}$ and $\phi_{k+1}^{\mathrm{II}} \geq \phi_{k}^{\mathrm{II}}$.

Following the determination of $\Phi^{\mathrm{I}}$, $\Phi^{\mathrm{II}}$, the DTW distance $\delta$ is calculated as follows:

$\delta=\sum_{k=1}^{K} \mathrm{D}\left(\mathbf{f}_{\phi_{k}^{\mathrm{I}}}^{\mathrm{I}}, \mathbf{f}_{\phi_{k}^{\mathrm{II}}}^{\mathrm{II}}\right)$.

\section{Comparison of Arm Postures, Penalizing Function}

The similarity of two samples (i.e. feature spaces) can be quantified by evaluating the penalizing function D $\left(\mathbf{f}_{i}^{\mathrm{I}}, \mathbf{f}_{j}^{\mathrm{II}}\right)$. As shown in (1), the feature vector of a sample contains the anatomic angles of the shoulder, elbow and wrist:

$$
\begin{aligned}
& \mathbf{f}_{i}^{\mathrm{I}}=\left(\alpha_{\mathrm{s}_{i}}^{\mathrm{I}}, \beta_{\mathrm{s}_{i}}^{\mathrm{I}}, \gamma_{\mathrm{s}_{i}}^{\mathrm{I}}, \beta_{\mathrm{e}_{i}}^{\mathrm{I}}, \alpha_{\mathrm{w}_{i}}^{\mathrm{I}}, \beta_{\mathrm{w}_{i}}^{\mathrm{I}}, \gamma_{\mathrm{w}_{i}}^{\mathrm{I}}\right)^{\mathrm{T}} \\
& \mathbf{f}_{j}^{\mathrm{II}}=\left(\alpha_{\mathrm{s}_{j}}^{\mathrm{II}}, \beta_{\mathrm{s}_{j}}^{\mathrm{II}}, \gamma_{\mathrm{s}_{j}}^{\mathrm{II}}, \beta_{\mathrm{e}_{j}}^{\mathrm{II}}, \alpha_{\mathrm{w}_{j}}^{\mathrm{II}}, \beta_{\mathrm{w}_{j}}^{\mathrm{II}}, \gamma_{\mathrm{w}_{j}}^{\mathrm{II}}\right)^{\mathrm{T}}
\end{aligned}
$$

The penalizing function is defined by

$$
\begin{aligned}
& \mathrm{D}\left(\mathbf{f}_{i}^{\mathrm{I}}, \mathbf{f}_{j}^{\mathrm{II}}\right)=\left|\mathbf{f}_{i}^{\mathrm{I}}-\mathbf{f}_{j}^{\mathrm{II}}\right|+P_{\mathrm{v}} \frac{1}{\tau} \mid\left(\mathbf{f}_{i+1}^{\mathrm{I}}-\mathbf{f}_{i}^{\mathrm{I}}\right)- \\
& -\left(\mathbf{f}_{j+1}^{\mathrm{II}}-\mathbf{f}_{j}^{\mathrm{II}}\right) \mid \\
& +P_{\mathrm{h}}\left(1-\frac{\mathbf{f}_{i}^{\mathrm{I}} \cdot \mathbf{f}_{j}^{\mathrm{II}}}{\left|\mathbf{f}_{i}^{\mathrm{I}}\right|\left|\mathbf{f}_{j}^{\mathrm{II}}\right|}\right)
\end{aligned}
$$

where $\tau$ is the sampling time, while $P_{\mathrm{v}}$ and $P_{\mathrm{h}}$ are weight parameters of the penalizing function for velocity and angle differences, respectively. The last term for the angle difference 
represents $1-\cos \in$, where $\in$ is the angle between the two feature vectors, which calculated by their scalar product.

In most of the automatic speech recognition applications the penalizing function consists only of the angle term, since the amplitude, i.e. the volume of the speech is not considered to increase or decrease the difference between two input sequences. Contrarily, in our case the feature vectors are containing the anatomic angles and/or anatomic positions of the human arm, therefore the Euclidean distances cannot be neglected, moreover, jerky motion (opposite to smoothly performed ADLs) has to be penalized by means of the term for velocities.

\section{Computing the optimal alignment}

In the first step, the calculation of the optimal alignment begins with the comparison of each sample $\mathbf{f}_{i}^{\mathrm{I}}$ to all other samples $\mathbf{f}_{j}^{\mathrm{II}}$, and the results obtained by the evaluations of the penalizing function are stored in matrix $M_{i j}=$ $\mathrm{D}\left(\mathbf{f}_{i}^{\mathrm{I}}, \mathbf{f}_{j}^{\mathrm{II}}\right)$. Due to the term of the velocity differences, the last samples in each data sets cannot be used in the first and third term of the penalizing function (9), $i \in[1, n-1]$, $j \in[1, m-1]$. Matrix $\mathbf{M}$ is illustrated in Figure 3 , in case of the comparison of two healthy subjects performing the same ADL (measured data shown in Figure 5, left column).
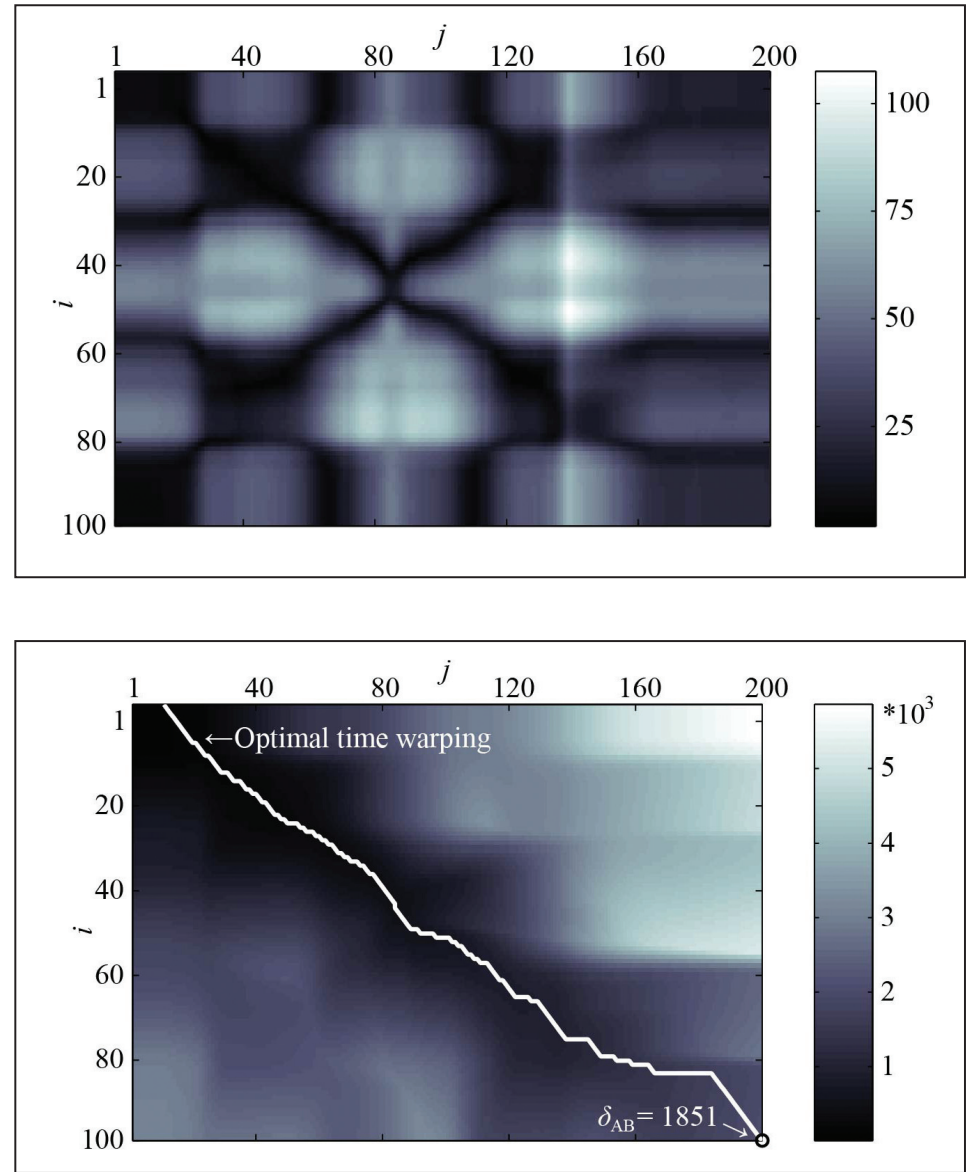

Figure 3.

Illustrating matrix $\mathbf{M}$, for the comparison of data shown in Figure 5, see colorbar on the left for the values of each matrix element

Figure 4.

Illustrating matrix $\mathbf{C}$ and the optimal time warping, in case of the comparison of data shown in Figure 5, see colorbar on the left for the values of each matrix element 
Following the calculation of matrix $\mathbf{M}$, the cumulated matrix $\mathbf{C}$ should be determined. Each element $C_{i j}$ of matrix $\mathbf{C}$ represents the least possible sum of those elements of $\mathbf{M}$, on which a step is taken when considering all the steps along the possible paths that connect elements $M_{11}$ and $M_{i j}$ fulfilling all the constraints listed in 3.1 including the monotonicity one (see Figure 4). The bottom right corner element of $\mathrm{C}$ defines the DTW distance $\delta$.

\section{Results}

\section{Comparison of ADL Performances}

Figure 5 and 6 illustrate the results of two DTW analyses. In Figure 5 the comparison of two healthy subjects (person $A$ solid line and person $B$ dashed line) is shown; both subjects are performing the same $\mathrm{ADL}$, opening and closing a window. As it can be seen from the

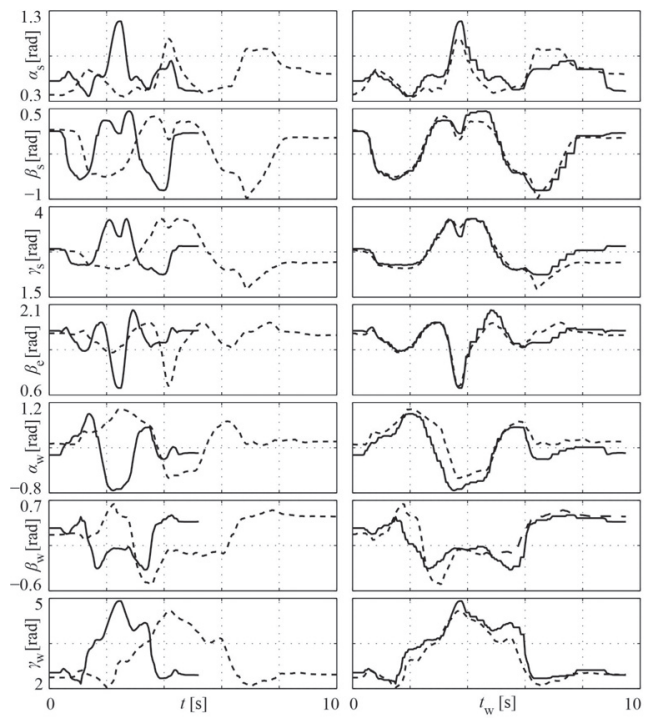

Figure 5. Comparison of two healthy ADL performances, person $A$ solid line and person $B$ dashed line, $\delta_{\mathrm{AB}}=1851$ original measurement data (shown in the left column), there is a significant difference in the paces of the functional movements. Person $A$ finishes in cca. 5 seconds, while person $\mathrm{B}$ needs cca. 10 seconds to complete the movement. The right column of the figure presents the aligned, that is the warped data in case of the optimal alignment (for penalizing parameters $P_{\mathrm{v}}=25$ and $P_{\mathrm{h}}=10$, the resulted DTW distance is $\delta_{\mathrm{AB}}=1851$ ).

The comparison of a healthy (person $A$, solid line, same as in Figure 5) and a stroke affected (person $C$, dashed line) subject's ADL performances is shown in Figure 6. Though person $C$ completes the function movement within roughly the time as person $B$, the DTW difference between $A$ and $C$ is significantly greater $\left(\delta_{\mathrm{AC}}=3923\right)$ than between $A$ and $B$, hence this difference is induced by the variance in the measured anatomic angles (note that $\left.\delta_{\mathrm{BC}}=2661\right)$.

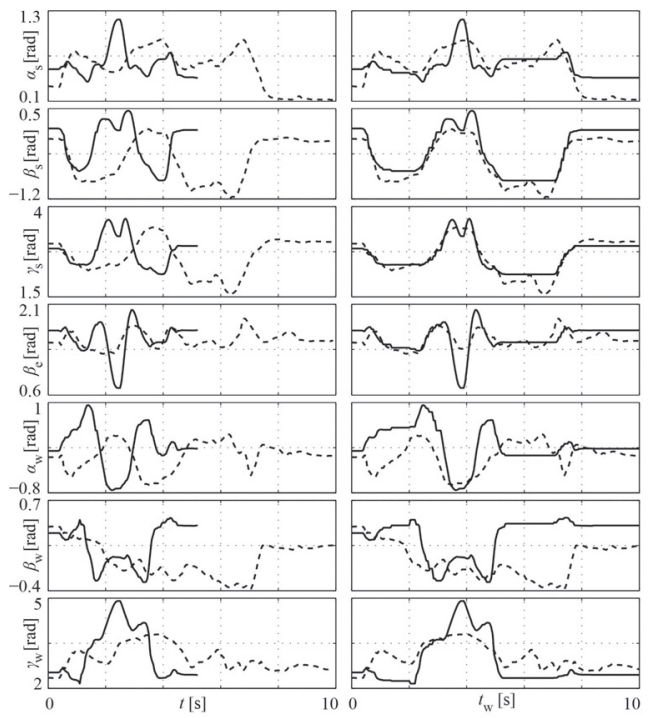

Figure 6. Comparison of a healthy and a stroke affected ADL performance, person $A$ solid line and person $C$ dashed line, $\delta_{\mathrm{AC}}=3923$ 


\section{Multidimensional Scaling Method}

Following the determination of the DTW distances for all possible dataset-pairs, multidimensional scaling (MDS) is a commonly used method for visualizing the level of similarity of individual cases. ${ }^{7}$ In this study, classical MDS method $^{8}$ is used. For a given distance matrix D that contains the pairwise differences of individual objects, MDS algorithm aims to place each object in an $N$-dimensional space such

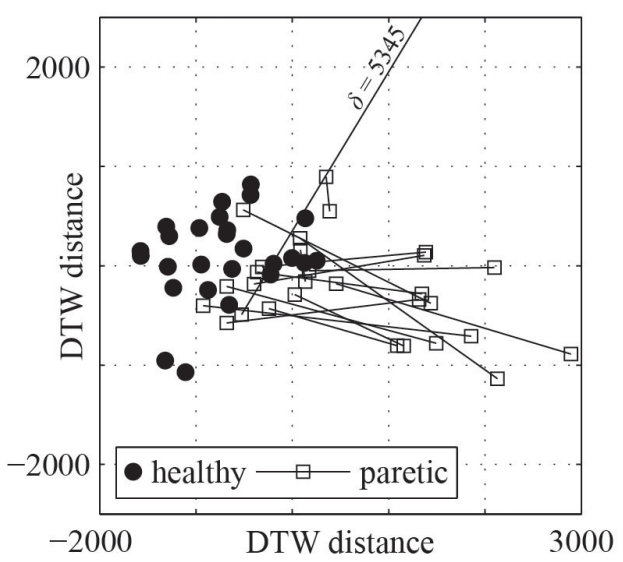

Figure 7. Scatter plot of ADL 1 performances (picking up a mug)

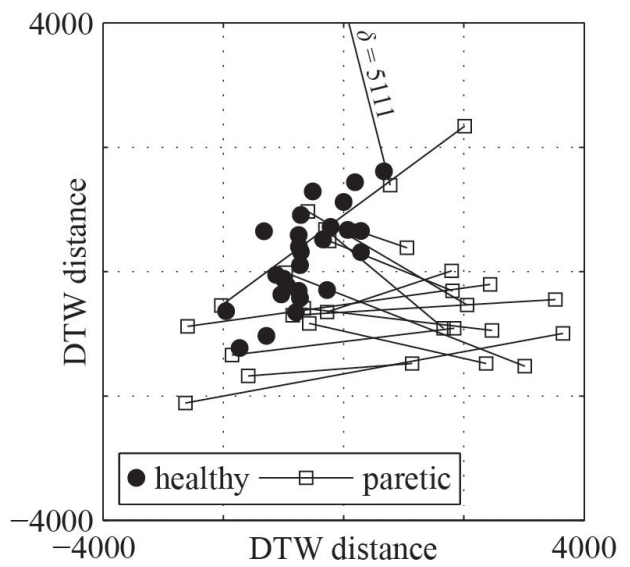

Figure 9. Scatter plot of ADL 3 performances (picking up a towel) that the between-object distances are preserved as well as possible. Therefore, the difference matrix does not need to be an Euclidean distance matrix.

\section{Scatter Plots of ADL Measurements}

Since the DTW algorithm can produce such pairwise distances, that does not even obey the triangle inequality, MDS method is needed to optimize object locations for a two-dimension-

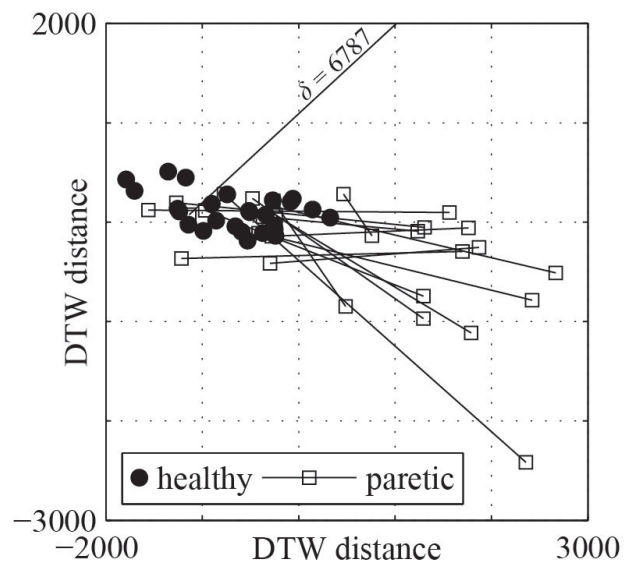

Figure 8. Scatter plot of ADL 2 performances (picking up a phone)

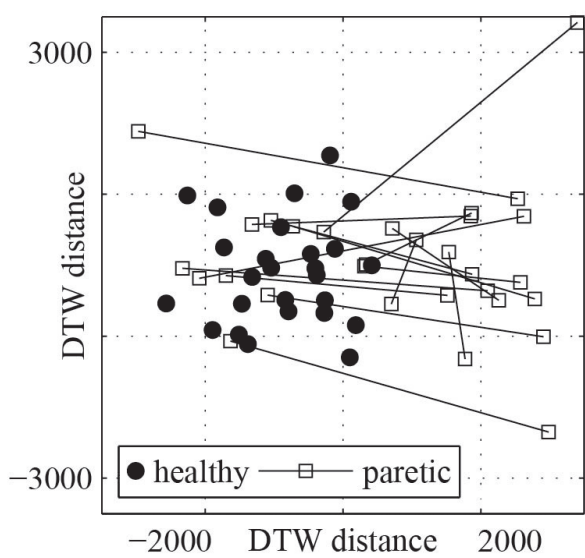

Figure 10. Scatter plot of ADL 4 performances (opening and closing a window) 


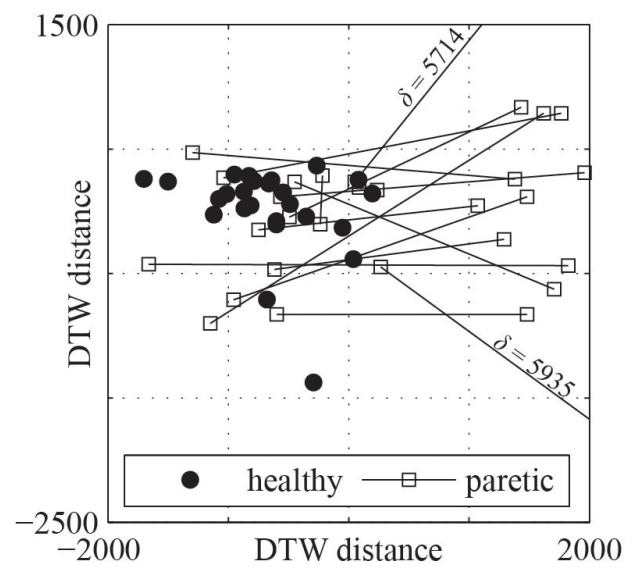

Figure 11. Scatter plot of ADL 5 performances (doing and undoing a zipper)

al scatter plot. These scatter plots are presented for all the 5 ADLs in Figures 7-11. In these figures solid discs represent healthy subjects, and squares represent the hemiparetic subjects, solid lines connect left and right hands of each stroke affected patient. By tuning $P_{\mathrm{v}}$ and $P_{\mathrm{h}}$ parameters in the penalizing function (9), two clusters had been evolved in the scatter plots. One contains the performances regarding both arms of healthy subjects and the nonparetic arm of hemiparetic subjects, and the other cluster is built up by the paretic arms of the stroke affected subjects.

\section{Conclusions}

According to the presented results, the performance of a post-stroke patient can be quantified as its distance from the cluster consisting of the reference $\mathrm{ADL}$ performances, i.e. the rehabilitation progress can be tracked by on objective, sensory based scale, lacking the possible errors due to human motion cognition. Due to the authors expectations, in the future standardized clinic scales, such as Fugl-Meyer, Asworth, Barthel, can be aided by DTW metrics. In order to achieve a good correlation with clinic scales, further evaluations are needed involving clinical professionals.

\section{REFERENCES}

1. Tormene P, Giorgino T, Quaglini S, Stefanelli M. Matching incom plete time series with dynamic time warping: an algorithm and an application to p ost-stroke rehabilitation. Artifi cial Intelligence in Medicine 2009;45:11-34.

2. Rath $T$, Manmatha $R$. Word image matching using dynamic time warping. Manmatha $\mathrm{R}$, editor. Proceedings of the IEEE computer society conference on computer vision and pattern recognition vol. 2; Los Alamitos, CA. USA: IEEE Computer So ciety; 2003. p. I I-521-7.

3. Tuzcu $V$, Nas $S$. Dynamic time warping as a novel to pattern recognition of ECG changes in heart rhythm disturbances. Jamsh idi M, Johnson M, Chen P, editors. Proceedings of the IEEE international conference on systems, man and cy- bernetics vol. 1; Los Alamitos, CA. USA: IEE E Computer Society; 2005. p. 182-6.

4. Brina CD, Niels $R$, Overvelde A, Levi $G$, Hulstijn $W$. Dynamic time warping: a new method in the study of poor handwriting. Human Movement Science 2008;27(2):242:55.

5. Wu H, Kido R, Shioyama T. Improvement of continuous dynamic programming for human gesture recognition. Proceedings of the 15 th international conference on pattern recognition vol. 2; Los Alamitos, CA. USA: IEEE Computer So ciety; 2000. p. 945.

6. Gollmer K, Posten C. Supervision of bioprocesses using a dynamic time warping algorithm. Control Engineering Practice 1996;4(9):1287:95. 
7. Borg I, Groenen P. Modern multidimensional scaling: theory and applications. 2nd ed. New York: Springer; 2005.
8. Seber GA. Multivariate observations. Hoboken, NJ: John Wiley and Sons, Inc., 1984.

This work was supported in part by the Agency for Science, Technology and Research, Singapore, under SERC Grant 0921490082 and the Media Development Authority, Singapore under NRF IDM004-005 Grants, project name COSMOSYS, and the Hungarian grant TÉT_08-SG-2010-0002.

\section{Bálint Magyar}

Budapest University of Technology and Economics, Faculty of Mechanical Engineering, Department of Applied Mechanics

H-1111 Budapest, Mû́egyetem rkp. 5.

Tel.: (+36) 1 463-1436 